\title{
An Immersed Boundary Method for Computational Simulation of Red Blood Cell in Poiseuille Flow
}

\author{
Reza ESMAILY*, Nader POURMAHMOUD**, Iraj MIRZAEE*** \\ *Department of Mechanical Engineering, Urmia University Pardis, Urmia, Iran, E-mail: reza_esmaily82@yahoo.com \\ **Department of Mechanical Engineering, Urmia University Pardis, Urmia, Iran, E-mail: n.pormahmod@urmia.ac.ir \\ ***Department of Mechanical Engineering, Urmia University Pardis, Urmia, Iran, E-mail: i.mirzaee@urmia.ac.ir \\ cross $^{\text {ref }}$ http://dx.doi.org/10.5755/j01.mech.24.3.18063
}

\section{Introduction}

Blood is a multiphase fluid that is primarily made of red blood cells (RBCs), white blood cells, and platelets suspended in plasma. Under normal, healthy conditions, a freely suspended RBC is a biconcave discoid with $8 \mu \mathrm{m}$ diameter and $2 \mu \mathrm{m}$ thickness. RBCs constitute; $40-45 \%$ of the total blood volume. Being highly deformable particles, RBCs can easily squeeze through the smallest capillaries having internal diameter less than their characteristic size. The particulate nature of the blood and the deformability of the RBCs determine the overall rheological behavior of the blood [1]. The lattice Boltzmann method (LBM) in combination with IBM has been used for simulating the motion and deformation of elastic bodies immersed in fluid flow including red blood cells (RBCs). Zhang et al. [2, 3] studied the dynamic behavior of $\mathrm{RBC}$ in shear flow and channel flow and investigated several hemodynamic and rheological properties, using a combination of LBM and IBM. Cheng et al. [4] have proposed a proper model to simulate the fast boundary movements and a high pressure gradient occurred in the fluid-solid interaction. In their research mitral valve jet flow considering the interaction of leaflets and fluid has been simulated. Alizadeh et al. [5, 6] investigated numerically the motion and deformation of a RBC in a viscous shear flow utilizing a combined LBM-IBM. Several recent numerical studies have focused on the behavior of deformable RBCs in microvascular flows [7-13]. RBC motions are well described by existing numerical techniques. Li et al. [14] applied the lattice Boltzmann method (LBM) to simulate two-dimensional rigid particle suspensions through a stenosed microvessel. Hyakutake et al. [15] conducted a two-dimensional simulation of the stenosed microvascular flow with rigid RBCs assuming primary pulmonary hypertension due to the stenosis of lung arteriole. Xu et al. [16] performed a two-dimensional simulation of RBC aggregation flow through a stenosed microvessel. There are few studies that focus on the time variation of RBC shape and the flow resistance in stenosed microvessels that have diameter less than $10 \mu \mathrm{m}$ [10]. Pozrikidis [17] have used boundary integral method to study motion and deformation of RBCs in the shear flow and the flow in the channel. Zhao et al. [18] have studied the time variations of RBCs deformation and flow resistance in the stenosed microvessels having a diameter less than $10 \mu \mathrm{m}$, using boundary integral method. Eggleton and Popel [19] combined immersed boundary method (IBM) with finite element method to simulate three-dimensional deformation of a RBC in a shear flow. Bagchi [1] has simulated a suspension containing multiple cells in the range of vessel size $20-30 \mu \mathrm{m}$ and discharge hematocrit 10-60\%, using IBM. Sun and Munn [20, 21] have studied RBC deformation in a $20-40 \mu \mathrm{m}$ channel using lattice Boltzmann method (LBM). They modeled the RBCs as two-dimensional solid particles. Li et al. [22] have used LBM for two-dimensional simulating of rigid particle suspensions in a stenosed microvessel. IBM is one of the methods that have been used successfully in recent decades to simulate the dynamics of flexible bodies in the fluid flow. This method was introduced for the first time in 1972 by Peskin [23] to study the flow around heart valves and developed as an efficient method to solve problems involving fluid-solid interactions. It is a combination of both the mathematical formulation and numerical scheme [24-26]. In this research, moving and deformation of Red Blood Cell(RBC) in microchannel with stenosis curve is investigated by Lattice Boltzmann method and immersed boundary. The results of this paper were compared to the available results and good agreements were observed.

\section{Governing equations}

The equations governing the combination of fluid and solid motions are as following:

$$
\begin{aligned}
& \rho\left(\frac{\partial \vec{u}}{\partial t}+\vec{u} \cdot \nabla \vec{u}\right)=-\nabla p+\eta \nabla^{2} \vec{u}+\vec{f}(\vec{x}, t) . \\
& \vec{f}(\vec{x}, t)=\int_{\Gamma} \vec{F}(s, t) \delta(\vec{x}-\vec{X}(s, t)) d s .
\end{aligned}
$$

The velocity of any point on the solid surface must be equal to that of the adjacent fluid particle, i.e.,

$$
\begin{aligned}
& \vec{U}(s, t)=\vec{u}(\vec{X}(s, t), t)=\frac{\partial \vec{x}}{\partial t}= \\
& =\int_{\Gamma} \vec{u}(\vec{x}, t) \delta(\vec{x}-\vec{X}(s, t)) d \vec{x}
\end{aligned}
$$

In the above equations, $\rho$ and $\eta$ are the mass density and dynamic viscosity of the fluid, respectively. In addition, $\vec{u}$ and $\rho$ indicate the velocity and pressure fields, respectively. The term $\vec{f}(\vec{x}, t)$ on the right-side of Eq. (1) denotes the RBC(Red Blood Cell) forces (tensile and bending) due to the elastic boundary immersed in the fluid.

Eq. (2) indicates that the force density of the fluid $\vec{f}(\vec{x}, t)$ is obtained from the force density of the RBC $\vec{F}(s, t)$. Equation (3) represents the no-slip condition at the 
fluid-solid interface, as the solid boundary moves with the same velocity as that of the surrounding fluid.

Mathematically the Dirac delta function $\delta(\vec{x})$ is discontinuous and has to be smoothed for numerical implementation:

$$
\delta(\vec{x})=\frac{1}{h^{2}} \Delta\left(\frac{x}{h}\right) \Delta\left(\frac{y}{h}\right),
$$

where:

$$
\Delta(r)=\left\{\begin{array}{cll}
\frac{1}{8}\left(3-2 r+\sqrt{1+4 r-4 r^{2}}\right) & \text { for } & 0 \leq r \leq 1 \\
\frac{1}{8}\left(5-2 r-\sqrt{-7+12 r-4 r^{2}}\right) & \text { for } & 1<r \leq 2, \\
0 & \text { for } 2<r
\end{array}\right.
$$

where: $h$ is the distance between two Eulerian grid points and $r$ denotes the distance between any two Eulerian and Lagrangian points.

In the IBM, to calculate the momentum exchange, the following collision function is used:

$$
f_{i}\left(\vec{x}+\vec{e}_{i} \Delta t, t\right)-f_{i}(\vec{x}, t)=-\frac{f_{i}(\vec{x}, t)-f_{i}^{e q}(\vec{x}, t)}{\tau}+\Delta t k_{i}
$$

where: $f_{i}(\vec{x}, t)$ is the density distribution function of particles with the velocity $\hat{e}_{i}$ located at position $\vec{x}$ at time $t . \Delta t$ is time step, $f_{i}^{e q}(\vec{x}, t)$ is the equilibrium distribution function, $\tau$ indicates the dimensionless relaxation time and $k_{i}$ denotes the body force associated with the immersed body. In the present research, the LBM with two-dimensional model of D2Q9 has been used.

The particle velocity in the corresponding nine directions can be written as follows:

$$
\hat{e}_{i}=\left\{\begin{array}{ll}
(i, i) & ; i=0 \\
\left(\cos \frac{\pi}{2}(i-1), \sin \frac{\pi}{2}(i-1)\right) c & ; i=1-4, \\
\sqrt{2}\left(\cos \frac{\pi}{2}\left(i-\frac{9}{2}\right), \sin \frac{\pi}{2}\left(i-\frac{9}{2}\right)\right) c & ; i=5-8
\end{array},\right.
$$

where: $c=\Delta x / \Delta t$. Here, $\Delta x$ is the distance between two successive nodes in the Euler grid. In the present research it is assumed that $\Delta x=\Delta t=1$. The equilibrium density distribution function is written as follow:

$$
f_{i}^{e q}(\vec{x}, t)=w_{i} \rho\left[1+3 \frac{\left(\hat{e}_{i} \cdot \vec{u}\right)}{c^{2}}+\frac{9}{2} \frac{\left(\hat{e}_{i} \cdot \vec{u}\right)^{2}}{c^{4}}-\frac{3}{2} \frac{|\vec{u}|^{2}}{c^{2}}\right] .
$$

The fluid pressure $\rho$ is calculated via an isothermal equation of state $\left(p=\rho C_{S}^{2}\right)$, where $C_{3}=c / \sqrt{3}$ is the speed of sound and $\rho$ is density. In addition, $w_{i}$ are weight coefficients with the following values:

$$
w_{i}=\left\{\begin{array}{ll}
\frac{4}{9} & ; \quad i=0 \\
\frac{1}{9} & ; \quad i=1-4 \\
\frac{1}{36} & ; \quad i=5-8
\end{array} .\right.
$$
$k_{i}$ is defined as:

$$
k_{i}=\left(1-\frac{1}{2 \tau}\right) w_{i}\left[\frac{3\left(\hat{e}_{i}-\vec{u}\right)}{c^{2}}+\frac{9\left(\hat{e}_{i} \cdot \vec{u}\right)}{c^{4}} \hat{e}_{i}\right] \cdot \vec{f} .
$$

In addition, the density and microscopic fluid velocity are calculated from the following relations,

$$
\begin{aligned}
& \rho=\sum_{i=0}^{8} f_{i} . \\
& \vec{u}=\frac{1}{\rho}\left[\sum_{i=0}^{8} f_{i} \hat{e}_{i}+\frac{\Delta t}{2} \vec{f}\right] .
\end{aligned}
$$

The Lagrangian force density $\vec{F}$ comprises two parts of tension-compression $\vec{F}_{S}$ and bending $\vec{F}_{b}$ forces, i.e.[27]:

$$
\vec{F}(s, t)=\vec{F}_{s}(s, t)+\vec{F}_{b}(s, t) .
$$

This force is related to the elastic potential energy density $\omega$ as follows (thanks to the virtual work theorem) :

$$
\vec{F}(s, t)=-\frac{\partial \omega}{\partial \vec{x}}=-\frac{\partial\left(\omega_{s}+\omega_{b}\right)}{\partial \vec{X}},
$$

where:

$$
\omega_{s}=\frac{1}{2} E_{s} \sum_{J=1}^{N-1}\left(\frac{\left|\vec{X}_{j+1}-\vec{X}_{j}\right|^{2}}{\Delta s}-1\right)^{2} \Delta s,
$$

and

$$
\omega_{b}=\frac{1}{2} E_{b} \sum_{J=2}^{N-1}\left(\frac{\left|\vec{X}_{j+1}-2 \vec{X}_{j}+\vec{X}_{j-1}\right|^{2}}{(\Delta s)^{4}}\right) \Delta s .
$$

Here: $E_{s}$ and $E_{b}$ are elastic modulus (tension/compression constant) and bending modulus, respectively.

The discretized form of Lagrangian force density $\vec{F}$, and elastic potential energy density will become: 


$$
\begin{aligned}
& \left.\left(\vec{F}_{s}\right)_{k}=\frac{E_{s}}{(\Delta s)^{2}} \sum_{j=1}^{N-1}\left\{\left|\vec{X}_{j+1}-\vec{X}_{j}\right|-\Delta s\right) \times \frac{\vec{X}_{j+1}-\vec{X}_{j}}{\left|\vec{X}_{j+1}-\vec{X}_{j}\right|}\left(\delta_{j, k}-\delta_{j+1, k}\right)\right\} . \\
& \left(\vec{F}_{b}\right)_{k}=\frac{E_{b}}{(\Delta s)^{4}} \sum_{j=2}^{N-1}\left\{\left(\vec{X}_{j+1}-2 \vec{X}_{j}+\vec{X}_{j-1}\right) \times\left(2 \delta_{j, k}-\delta_{j+1, k}-\delta_{j-1, k}\right)\right\} .
\end{aligned}
$$

In Eqs. (16-17) $k=1,2, \ldots, N$. ( $N$ is the total number of Lagrangian nodes on the RBC), $\left(\vec{F}_{S}\right)_{k}$ and $\left(\vec{F}_{b}\right)_{k}$ are elastic Lagrangian forces associated with the node $k$ on the RBC and $\delta_{j, k}$ is the Kronecker delta function.

When the Lagrangian forces on the RBC are calculated, all the translational and rotational speeds are updated explicitly. It should be noted that the solid RBC moves continuously based on Newtonian dynamics and finally the new position of the membrane is obtained.

In the present work, the blood is considered as Newtonian fluids. The Newtonian nature of these fluids has been well estimated [28]. The non-Newtonian behaviour of blood is mainly due to the deformation of RBCs [1]. The Re number and other dimensionless parameters are defined as follows:

$$
R e=\frac{\rho u_{\max } H}{\mu} ; x=\frac{x^{*}}{D} ; y=\frac{y^{*}}{D} .
$$

Where $x$ and $y$ denote respectively the dimensionless vertical coordinate and horizontal coordinate. In addition, $D$ and $u_{\max }$ are the height of microchannel and the maximum velocity of the Poiseuille flow, respectively. The purpose of this study is to investigate the ability of the LatticeBoltzmann and the immersed boundary method to model the deformation of flexible membranes, red blood cells, and etc, inside the flow.

Innovation of present work is investigating of the hardness of the red blood cell membrane on its deformation and the flow velocity of a microchannel with curved stenosis using the Lattice-Boltzmann and the immersed boundary method Also, in this work.

\section{Validation}

In order to compare the present results with other works, the results are presented in the forms of average drag coefficient, maximum lift coefficient and Struhall number for $R e=100$ in Table 1 .

The comparison of average drag coefficient, maximum lift coefficient and Struhall number for one cylinder for $\operatorname{Re}=100$

\begin{tabular}{|c|c|c|c|}
\hline & $\begin{array}{c}\text { average } \\
\text { drag coef- } \\
\text { ficient }\end{array}$ & $\begin{array}{c}\text { maximum lift } \\
\text { coefficient }\end{array}$ & $\begin{array}{c}\text { Strouhal } \\
\text { number }\end{array}$ \\
\hline present work & 1.34 & 0.35 & 0.165 \\
\hline Kang [29] & 1.33 & 0.32 & 0.165 \\
\hline Liu et al. [30] & 1.37 & 0.33 & 0.165 \\
\hline
\end{tabular}

Considering the data in Table 1 , one can see that the present results are in good agreement with the other results. In these references, the governing equations (NavierStokes equation) are resolved with an unstructured Spectral Element Method. In the present work. The circular cylinder has been considered in the form of an immersed boundary with a large elastic property in the flow. The Bounce back boundary condition has been used in order to satisfy no-slip boundary condition on the cylinder surface.

\section{Results and discussion}

As can be seen from Fig. 1, a RBC at a specific distance from the entrance of microchannel is in the poiseuille flow, stenosis of microchannel in this problem has curved shape which help the problem to be real. In this research, the effects of flexibility of cells is examined. First, deformation of healthy cells with a coefficient of tensile and bending $6 \times 10^{-6} \mathrm{~N} / \mathrm{m}$ and $2 \times 10^{-19} \mathrm{~N}$. m, respectively and then behaviour of sick RBC with a coefficient of tensile and bending $6 \times 10^{-2} \mathrm{~N} / \mathrm{m}$ and $5 \times 10^{-18} \mathrm{~N}$. m, respectively. Healthy RBC has more flexibility, thereby it easily passed the stenosis which is important in some blood diseases. In Figs. 2 and 3 since the velocity of blood flow is increased in the stenosis part, more deformations of the RBC would take place at this section and the Reynolds number is considered to be $R e=0.35$.

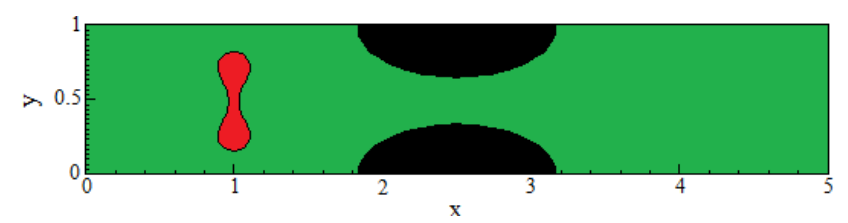

Fig. 1 Geometry and nomenclature of the microchannel with stenosis

As can be seen(Fig.4), healthy RBC due to more flexibilty has more deformation and it stretch more in the longitudinal direction of microchannel and more shear force from the fluid exerted on the surface of the cell. Healthy RBC needs more time to return to its original shape and need less energy to pass the stenosis. However, when the sick RBC reaches the stenosis, slightly reduces the effective cross-sectional area of the flow behind it due to its high hardness. In fact, it blocks the flow path more than the normal RBC. This would cause a decrease in the flow velocity and hence an increase in the flow pressure behind the RBC. In Fig. 5, flow velocity profile at $x=2.5$ can be observed ( $d$ is height of stensios). In flow without RBC, velocity profile is parabolic. The more the flexibility of $\mathrm{RBC}$ reduced, the velocity profile will be more flat. The sick RBC has less interaction with its surrounding fluid and the velocity of the plasma flow is low compared to the other cases [1]. This deceleration is with increase of pressure around the RBC. This pressure increase due to stiffness of RBC membrane can be seen in anemia and heart diseases. The translational speed of a RBC decreases as its elasticity modulus increases [31]. 


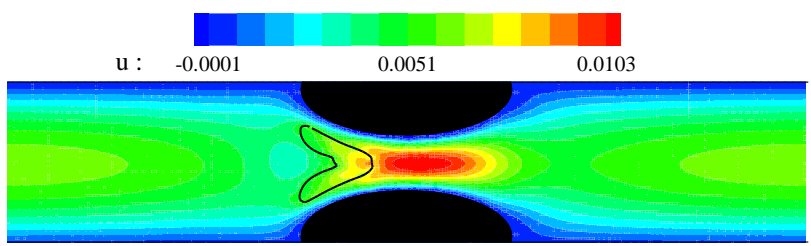

a

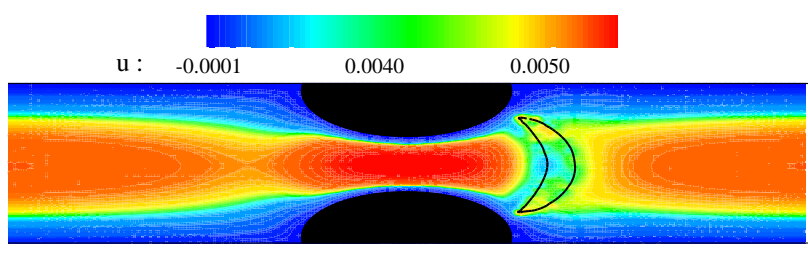

C

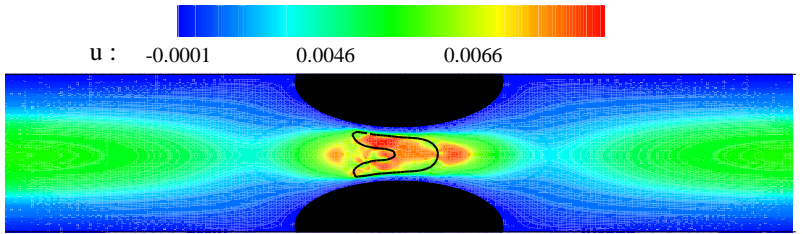

$\mathrm{b}$

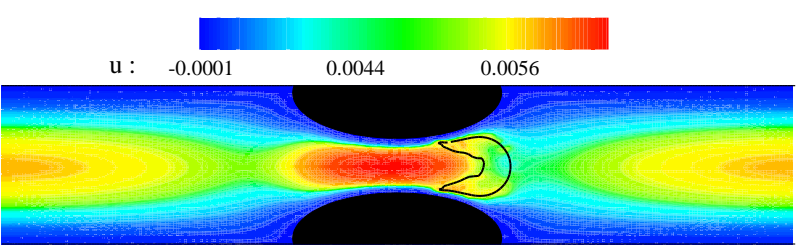

d

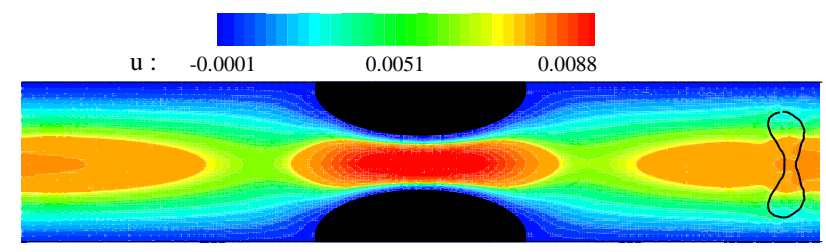

e

Fig. 2 Motion and deformation of a healthy (high deformable) RBC through a microchannel with stenosis, at the moment of time: a - $0.1 \mathrm{~ms}, \mathrm{~b}-0.2 \mathrm{~ms}, \mathrm{c}-0.3 \mathrm{~ms}, \mathrm{~d}-0.4 \mathrm{~ms}$ and e $-0.5 \mathrm{~ms}$

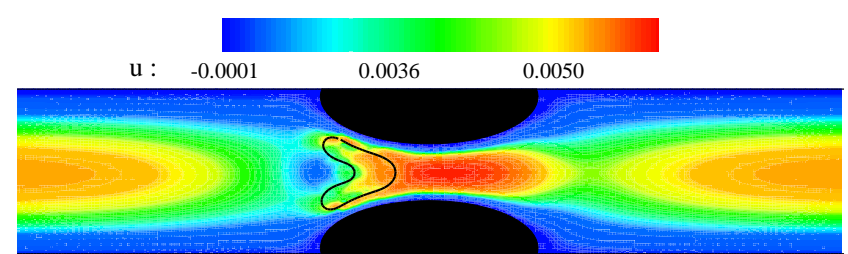

$\mathrm{a}$

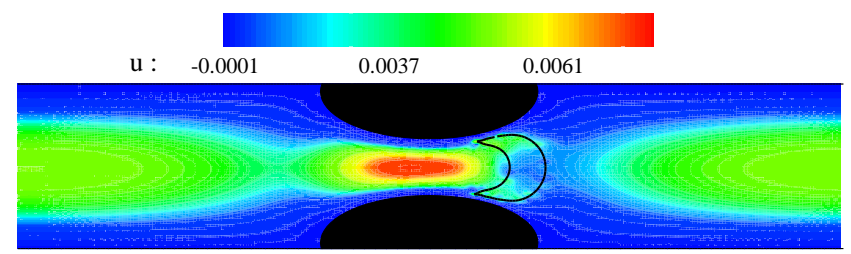

c

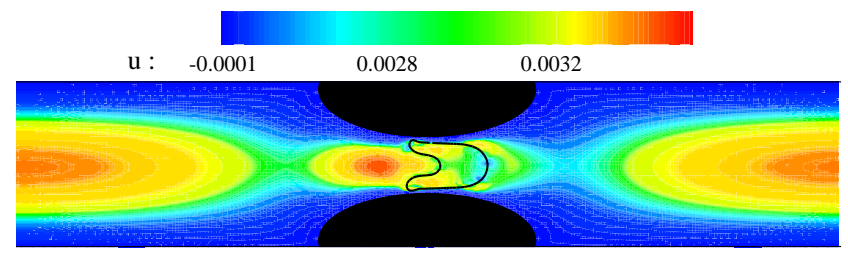

$\mathrm{b}$

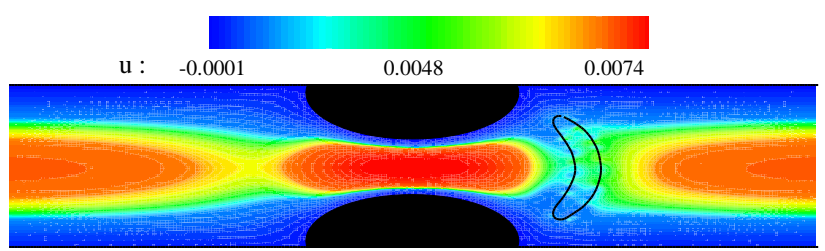

d

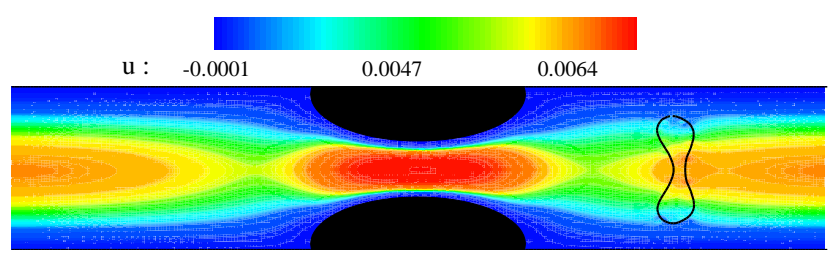

e

Fig. 3 Motion and deformation of a sick (low deformable) RBC through a microchannel with stenosis, at the moment of time: $\mathrm{a}-0.15 \mathrm{~ms}, \mathrm{~b}-0.25 \mathrm{~ms}, \mathrm{c}-0.35 \mathrm{~ms}, \mathrm{~d}-0.45 \mathrm{~ms}$ and $\mathrm{e}-0.55 \mathrm{~ms}$ 


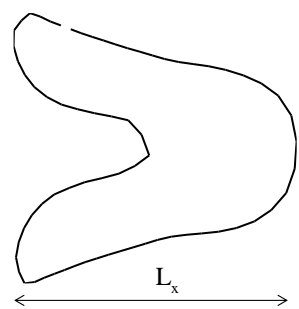

a

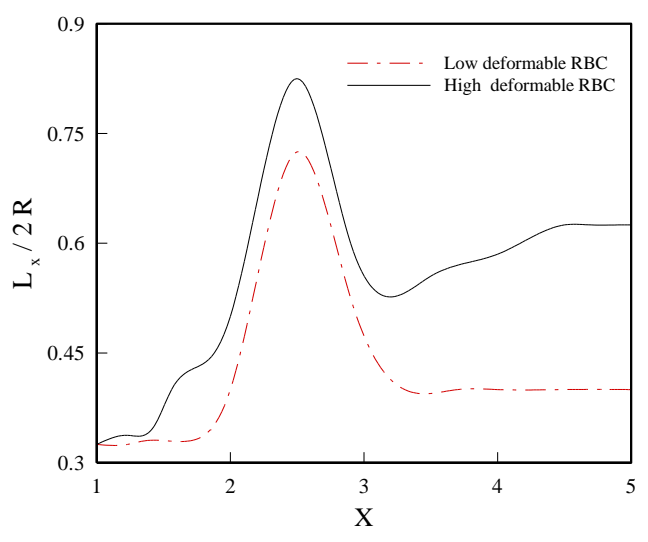

b

Fig. 4 Variations of parameter characterizing the deformation of a RBC. Parameter $L_{x}-(\mathrm{a})$, variations of $L_{x} / 2 R-($ b) versus the microchannel length

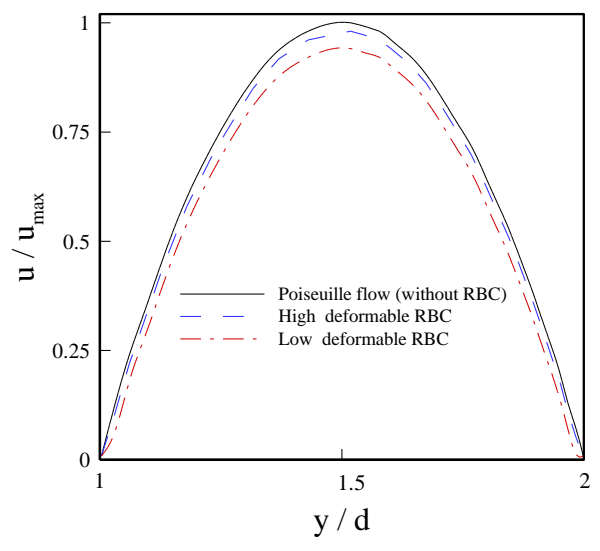

Fig. 5 x-component velocity profile

\section{Conclusions}

A hybrid LBM-IBM is used to simulate the hydrodynamic interaction of RBCs having different elastic moduli in a stenosed microchannel. The RBCs are considered as elastic boundaries immersed in the fluid flow and are represented in Lagrangian coordinates. The coupling method between the fluid and solid membranes is based on the IBM, which uses a uniform and fixed Eulerian mesh and removes the burden of expensive mesh updating in the traditional Arbitrary Lagrangian Eulerian approach. The results were found to be in good agreement with available data. It was shown that healthy RBC moves faster than the sick one. Velocity decrease due to existance of RBC with low flexibility results in increase of pressure around the RBC. This pressure increase due to stiffness of RBC membrane can be seen in anemia and heart diseases.

In many pathological conditions such as heart disease, blood pressure, anemia, malaria and ... flexibility of red blood cells reduced. Changes in the mechanical properties of red blood cells with disorders happened in the microscale system, such as capillaries and small veins, and sequently, it disorders the functioning of vital organs such as the brain and the kidneys. The determination of hemodynamic changes is a major step in the development of new methods for the diagnosis and treatment of these diseases. Understanding the biological system and examining various parameters affecting the flow of fluid caused by the immersed object can lead to the design of medical devices.

\section{References}

1. Bagchi, P. 2007. Mesoscale simulation of blood flow in small vessels, Biophysical journal 92: 858-877. https://doi.org/10.1529/biophysj.106.095042.

2. Zhang, J. F.; Johnson, P. C.; Popel, A.S. 2007. An immersed boundary lattice Boltzmann approach to simulate deformable liquid capsules and its application to microscopic blood flows, Physical biology 4: 285-295. https://doi.org/10.1088/1478-3975/4/4/005.

3. Zhang, J. F.; Johnson, P. C.; Popel, A. S. 2008. Red blood cell aggregation and dissociation in shear flows simulated by lattice Boltzmann method, Journal of biomechanics 41: 47-55.

https://doi.org/10.1016/j.jbiomech.2007.07.020.

4. Cheng, Y.; Zhang, H. 2010. Immersed boundary method and lattice Boltzmann method coupled FSI simulation of mitral leaflet flow, Computers \&Fluids 39:871-881.

https://doi.org/10.1016/j.compfluid.2010.01.003.

5. Alizadeh, A.; Dadvand, A. 2016. Simulation of the motion of two elastic membranes in Poiseuille shear flow via a combined immersed boundary-lattice Boltzmann method, Journal of Computational Science12:51-61. https://doi.org/10.1016/j.jocs.2015.11.008.

6. Alizadeh, A.; Dadvand, A. 2018. Hydrodynamic interaction of elastic membranes in a stenosed microchanne, Applied Mathematical Modelling 54: 361-377. https://doi.org/10.1016/j.apm.2017.09.042.

7. Boryczko, K.; Dzwinel, W.; Yuen, D. A. 2003. Dynamical clustering of red blood cells in capillary vessels, Journal of Molecular Modeling 9 :16-33. http://dx.doi.org/10.1007/s00894-002-0105-x.

8. Dupin, M. M.; Halliday, I.; Care, C. M.; Alboul, L.; Munn, L. L. 2007. Modeling the flow of dense suspensions of deformable particles in three dimensions, Physical Review E 75: 066707. https://doi.org/10.1103/PhysRevE.75.066707.

9. Sugiyama, K.; Ii, S.; Takeuchi, S.; Takagi, S.; Matsumoto, Y. 2010. Full Eulerian simulations of biconcave neo-Hookean particles in a Poiseuille flow, Computational Mechanics 46: 147-157. https://doi.org/10.1007/s00466-010-0484-2.

10. Zhao, H.; Isfahani, Amir.; Olson, A. H. G.; Freund, J. B. 2010. A spectral boundary integral method for flowing blood cells, Journal of ComputationalPhysics 229:3726-3744. https://doi.org/10.1016/j.jcp.2010.01.024.

11.Secomb, T. W. 2011.Mechanics and computational simulation of blood flow in microvessels, Medical Engineering \& Physics 33:800-804.

https://doi.org/10.1016/j.medengphy.2010.09.016.

12. Shi, L.; Pan, T.; Glowinski, R. 2012. Deformation of a 
single blood cell in bounded Poiseuille flows, Physical Review E 85:016307.

https://doi.org/10.1103/PhysRevE.85.016307.

13. Alizadehred, D.; Imai, Y.; Nakaaki, K.; Ishikawa, T.; Yamaguchi, Y. 2012. Quantification of red blood cell deformation at high-hematocrit blood flow in microvessels, Journal of Biomechanics 45:2684-2689. https://doi.org/10.1016/j.jbiomech.2012.08.026.

14. Li, H.; Fang, H.; Lin, Z.; Xu, S.; Chen, S. 2004. Lattice Boltzmann simulation on particle suspensions in a two-dimensional symmetric stenotic artery, Physical Review E 69: 031919. https://doi.org/10.1103/PhysRevE.69.031919.

15. Hyakutake, T.; Ohkawa, S.; Mohri, S.; Yanase, S. 2008. Lattice Boltzmann analysis of microvascular constriction flow including red blood cell and liposome-encapsulated hemoglobin, Theoretical Applied Mechanics Japan 56:215224. https://doi.org/10.11345/nctam.56.215.

16. Xu, Y.; Tian, F.; Deng, Y. 2013. An efficient red blood cell model in the frame of IB-LBM and its application,International Journal of Biomathematics6:1250061. https://doi.org/10.1142/S1793524512500611.

17. Pozrikidis, C. 1995. Finite deformation of liquid capsules enclosed by elastic membranes in simple shear flow, Journal of Fluid Mechanics 297: 123-152. https://doi.org/10.1017/S002211209500303.X.

18. Zhao, H.; Isfahani, Amir.; Olson, A.H.G.; Freund, J.B. 2010. A spectral boundary integral method for flowing blood cells, Journal of ComputationalPhysics229:3726-3744. https://doi.org/10.1016/j.jcp.2010.01.024.

19. Eggleton, C.D.;Popel, A.S.1998. Large deformation of red blood cell ghosts in a simple shear flow, Physics of fluids 10: 1834-1845.

https://doi.org/10.1063/1.869703.

20. Sun, C.; Migliorini, C.; Munn, L. L. 2003. Red blood cells initiate leukocyte rolling in post capillary expansions: a lattice Boltzmann analysis, Biophysical Journal 85:208-222. https://doi.org/10.1016/S0006-3495(03)74467-1.

21.Sun, C.; Munn, L.L. 2005. Particulate nature of blood determines macroscopic rheology: A 2-D lattice Boltzmann analysis, Biophysical Journal 88:1635-1645. https://doi.org/10.15299biophysj.104.051151.

22. Li, H. ; Fang, H. ; Lin, Z.; Xu, S.; Chen, S. 2004. Lattice Boltzmann simulation on particle suspensions in a two-dimensional symmetric stenotic artery, Physical review E 69: 031919. https://doi.org/10.1103/PhysRevE.69.031919.

23. Peskin, C. S. 1973. Flow patterns around heart valves: a digital computer method for solving the equations of motion, IEEE Transactions on Biomedical Engineering 20: 316-317. https://doi.org/ 10.1109/TBME.1973.324206.

24. Kim, J.; Kim, D.; Choi, H. 2001.An immersed-boundary finite-volume method for simulations of flow in complex geometries, Journal of Computational Physics 171:132-150. https://doi.org/10.1006/jcph.2001.6778.

25. Taira, K.; Colonius, T. 2007. The immersed boundary method: a projection approach, Journal of Computational Physics 225: 2118-2137. https://doi.org/10.1016/j.jcp.2007.03.005.

26. Pinelli, A.; Naqavi, I. Z.; Piomelli, U.; Favier, J. 2010. Immersed-boundary methods for general finite-difference and finite-volume Navier-Stokes solvers, Journal of Computational Physics 229: 9073-9091. https://doi.org/10.1016/j.jcp.2010.08.021.

27. Zhu, L.; He, G.;Wang, S.; Miller, L.; Zhang, X.; You, Q.; Fang, S. 2011.An immersed boundary method based on the lattice Boltzmann approach in three dimensions with application, Computers \& Mathematics with Applications 61: 3506-3518. https://doi.org/10.1016/j.camwa.2010.03.022.

28. Fung, Y.C. 2013. Biomechanics: Mechanical Properties of Living Tissues, Springer Science \& BusinessMedia. https://doi.org/10.1007/978-1-4757-2257-4.

29. Kang, S. 2003. Characteristics of flow over two circular cylinders in a side-by-side arrangement at low Reynolds numbers, Physics of Fluids 15: 2486-2498. https://doi.org/10.1063/1.1596412.

30. Liu, Kun.; Dong, M.A.; Sun, D. 2007. Wake patterns of flow past a pair of circular cylinders in side-by-side arrangements at low Reynolds numbers, Journal of Hydrodynamics 19: 690-697. https://doi.org/10.1016/S1001-6058(08)60005-0.

31. Xiong, W.; Zhang, J. 2010. Shear stress variation induced by red blood cell motion in microvessel, Annals of biomedical engineering 38: 2649-2659. https://doi.org/10.1007/s10439-010-0017-3.

R. Esmaily, N. Pourmahmoud, I. Mirzaee

\section{AN IMMERSED BOUNDARY METHOD FOR COMPUTATIONAL SIMULATION OF RED BLOOD CELL IN POISEUILLE FLOW}

S u m m a r y

In this paper, the effects of RBC hardness in a viscous incompressible flow is investigated. The effects of hardness changes on the membrane behavior and its around flow is studied. In the Immersed Boundary method the elastic membrane is modeled in Lagrangian coordinate but the flow field is discretized using a uniform and fixed Eulerian grid. When RBCs have less elastic properties; they pass the obstacle hardly and increase the pressure of around flow. Velocity decrease due to existance of RBC with low flexibility results in increase of pressure around the RBC. This pressure increase due to stiffness of RBC membrane can be seen in anemia and heart diseases. The translational speed of a RBC decreases as its elasticity modulus increases The comparison between the present results and other available results show that the Lattice Boltzmann and Immersed Boundary methods have good capability for modeling of immersed objects motions.

Keywords: red blood cell, Poiseuille flow, immersed boundary method, lattice Boltzmann method, elasticity modulus.

Received June 27, 2017

Accepted June 14, 2018 